\title{
Some Results in Stability Analysis of Hybrid Dynamical Systems
}

I.L.D. SANTOS 1 , G.N. SILVA ${ }^{2}$, Departamento de Ciências de Computação e Estatística, IBILCE, UNESP, 15054-000 São José do Rio Preto, SP, Brasil.

\begin{abstract}
In this paper we introduced a general model for the Hybrid Dynamical Systems and for such systems we introduced the usual concept of Lyapunov stability. Furthermore, we established two Principal Lyapunov Theorems and a converse theorem.
\end{abstract}

Keywords. Hybrid dynamical systems, Lyapunov stability, discontinuous dynamical systems.

\section{Introduction}

Hybrid systems are capable of exhibiting simultaneously several kinds of dynamic behavior, such that continuous-time dynamics, discrete-time dynamics, logic commands, and so forth.

At the present time, there does not appear to exist a satisfactory general model for hybrid dynamical systems which is situable for the qualitative analysis of such systems.

In the present paper we give a definition of hybrid dynamical system which covers a very large number of classes of hybrid systems and which is suitable for the qualitative analysis of such systems.

\section{Hybrid Systems}

\subsection{Hybrid dynamical systems}

Definition 2.1. Given the set $X, \prec \subset X \times X$ it is an order relationship in $X$ if for any $x, y, z \in X$ such that $(x, y) \in \prec \Leftrightarrow x \prec y$ :

(i) $x \prec x$;

(ii) if $x \prec y$ and $y \prec x$ then $x=y$;

(iii) if $x \prec y$ and $y \prec z$ then $x \prec z$.

Definition 2.2. We say that a function $\phi \in C\left[[0, r], \mathbb{R}^{+}\right]\left(\right.$respectively $\left.\phi \in C\left[\mathbb{R}^{+}, \mathbb{R}^{+}\right]\right)$ belongs to class $K(\phi \in K)$, if $\phi(0)=0$ and if $\phi$ is strictly increasing on $[0, r]$ (respectively on $\left.\mathbb{R}^{+}\right)$.

\footnotetext{
${ }^{1}$ iguerluis@hotmail.com (financial support CAPES).

${ }^{2}$ gsilva@ibilce.unesp.br
} 
Furthermore, we say that a function $\phi \in K$ defined on $\mathbb{R}^{+}$belongs to class $K \mathbb{R}$ if $\lim _{r \rightarrow \infty} \phi(r)=+\infty$.

Definition 2.3. A metric space $(T, \rho)$ is called a time space if:

(i) $T$ is completely ordered with order $\prec$;

(ii) $T$ has a minimal element $t_{\text {min }} \in T$, that is, for any $t \in T$ and $t \neq t_{\text {min }}$, it is true that $t_{\text {min }} \prec t$;

(iii) for any $t_{1}, t_{2}, t_{3} \in T$ such that $t_{1} \prec t_{2} \prec t_{3}$, it is true that $\rho\left(t_{1}, t_{3}\right)=\rho\left(t_{1}, t_{2}\right)+$ $\rho\left(t_{2}, t_{3}\right)$;

(iv) $T$ is unbounded from above, that is, for any $M>0$, there exists a $t \in T$ such that $\rho\left(t, t_{\min }\right)>M$.

Definition 2.4. Let $(X, d)$ be a metric space and let $A \subset X$. Let $(T, \rho)$ be a time space, and let $T_{0} \subset T$. For any fixed $a \in A, t_{0} \in T_{0}$, we call a mapping $p\left(., a, t_{0}\right): T_{a, t_{0}} \rightarrow X$ a motion if $p\left(t_{0}, a, t_{0}\right)=a$, where $T_{a, t_{0}}=\left\{t \in T: t_{0} \preceq t\right\}$.

Thus, we define hybrid dynamical systems:

Definition 2.5. Let $S$ be a set of motions, that is, $S \subset\left\{p\left(., a, t_{0}\right) \in \Lambda: p\left(t_{0}, a, t_{0}\right)=\right.$ $a\}$, where $\Lambda=\cup_{\left(a, t_{0}\right) \in\left(A \times T_{0}\right)}\left\{T_{a, t_{0}} \rightarrow X\right\}$. The five-tuple $\left\{T, X, A, S, T_{0}\right\}$ is called a hybrid dynamical system.

\subsection{Some qualitative characterizations}

Definition 2.6. Let $\left\{T, X, A, S, T_{0}\right\}$ be a hybrid dynamical system. A set $M \subset A$ is said to be invariant with respect to system $S$ (that is, $(S, M)$ is invariant) if a $\in M$ implies that $p\left(t, a, t_{0}\right) \in M$ for all $t \in T_{a, t_{0}}$ and all $t_{0} \in T_{0}$ such that $p\left(., a, t_{0}\right) \in S$.

Definition 2.7. We call $x_{0} \in A$ an equilibrium of a hybrid dynamical system $\left\{T, X, A, S, T_{0}\right\}$ if $\left(S,\left\{x_{0}\right\}\right)$ is invariant.

Definition 2.8. (Lyapunov Stability) Let $\left\{T, X, A, S, T_{0}\right\}$ be a hybrid dynamical system and let $M \subset A$ be an invariant set of $S$.

(i) We say that $(S, M)$ is stable if for every $\epsilon>0$, and $t_{0} \in T_{0}$ there exists a $\delta=$ $\delta\left(\epsilon, t_{0}\right)>0$ such that $d\left(p\left(t, a, t_{0}\right), M\right)<\epsilon$ for all $t \in T_{a, t_{0}}$ and for all $p\left(., a, t_{0}\right) \in S$, whenever $d(a, M)<\delta$.

(ii) We say that $(S, M)$ is uniformly stable if $\delta=\delta(\epsilon)$.

(iii) If $(S, M)$ is stable and if for any $t_{0} \in T_{0}$, there exists an $\eta=\eta\left(t_{0}\right)>0$ such that, for every $\epsilon>0$, there exists a $t_{\epsilon} \in T$ such that $d\left(p\left(t, a, t_{0}\right), M\right)<\epsilon$ whenever $t \in T$ and $t_{\epsilon} \preceq t$, for all $p\left(., a, t_{0}\right) \in S$ whenever $d(a, M)<\eta$, then $(S, M)$ is called asymptotically stable.

(iv) We call $(S, M)$ uniformly asymptotically stable if $(S, M)$ is uniformly stable and if there exists $a \delta>0$ and for every $\epsilon>0$ there exists $a \tau=\tau(\epsilon)>0$ such that $d\left(p\left(t, a, t_{0}\right), M\right)<\epsilon$ for all $t \in\left\{t \in T_{a, t_{0}}: \rho\left(t, t_{0}\right) \geq \tau\right\}$, and all $p\left(., a, t_{0}\right) \in S$ whenever $d(a, M)<\delta$.

$(v)(S, M)$ is said to be exponentially stable if there exists $\alpha>0$ such that for every $\epsilon>0$ and $t_{0} \in T_{0}$, there exists a $\delta=\delta(\epsilon)>0$ such that $d\left(p\left(t, a, t_{0}\right), M\right)<\epsilon e^{-\alpha \rho\left(t, t_{0}\right)}$ for all $t \in T_{a, t_{0}}$ and for all $p\left(., a, t_{0}\right) \in S$, whenever $d(a, M)<\delta$. 
Observation : The exponential stability of $(S, M)$ implies the uniform asymptotic stability of $(S, M)$.

Indeed, if $(S, M)$ is exponentially stable then for every $\epsilon>0$ and $t_{0} \in T_{0}$, there exists $\alpha>0$ and $\delta=\delta(\epsilon)>0$ such that $d\left(p\left(t, a, t_{0}\right), M\right)<\epsilon e^{-\alpha \rho\left(t, t_{0}\right)}$ for all $t \in$ $T_{a, t_{0}}$ and for all $p\left(., a, t_{0}\right) \in S$, whenever $d(a, M)<\delta$, therefore $d\left(p\left(t, a, t_{0}\right), M\right)<$ $\epsilon e^{-\alpha \rho\left(t, t_{0}\right)} \leq \epsilon$ and $(S, M)$ is uniformly stable.

Furthermore, for all $t \in T_{a, t_{0}}$ it is had $d\left(p\left(t, a, t_{0}\right), M\right)<\epsilon e^{-\alpha \rho\left(t, t_{0}\right)}$, thus, for any $\tau=\tau(\epsilon)>0$ such that $t \in\left\{t \in T_{a, t_{0}}: \rho\left(t, t_{0}\right) \geq \tau\right\}, d\left(p\left(t, a, t_{0}\right), M\right)<\epsilon e^{-\alpha \rho\left(t, t_{0}\right)}<$ $\epsilon$ and then $(S, M)$ is uniformly asymptotically stable.

\subsection{Embedding of hybrid dynamical systems into dynamical systems defined on $\mathbb{R}^{+}$}

Any time space $T$ can be embedded into the real space $\mathbb{R}^{+}$by means of a mapping $g: T \rightarrow \mathbb{R}^{+}$, having the following properties:

(i) $g\left(t_{\min }\right)=0$, where $t_{\text {min }}$ denotes the minimum element in $T$;

(ii) $g(t)=\rho\left(t, t_{\text {min }}\right)$ for $t \neq t_{\text {min }}$.

If we let $R_{1}=g(T)$, then $g$ is an isometric mapping from $T$ to $R_{1}$. Indeed, given $r \in R_{1}$, there exists $t \in T$ such that $r=g(t)$ and if $g\left(t_{1}\right)=g\left(t_{2}\right)$, for $t_{1} \prec t_{2}$, since $\rho\left(t_{\text {min }}, t_{2}\right)=\rho\left(t_{\text {min }}, t_{1}\right)+\rho\left(t_{1}, t_{2}\right)$ it follows that $g\left(t_{2}\right)-g\left(t_{1}\right)=\rho\left(t_{1}, t_{2}\right)$ and then $t_{1}=t_{2}$. Thus, $g$ is a bijection from $T$ onto $R_{1}$, furthermore, for $t_{1} \prec t_{2}$ it is had $d\left(g\left(t_{1}\right), g\left(t_{2}\right)\right)=\left|g\left(t_{2}\right)-g\left(t_{1}\right)\right|=\left|\rho\left(t_{1}, t_{2}\right)\right|=\rho\left(t_{1}, t_{2}\right)$, therefore $g$ is an isometric mapping from $T$ to $R_{1}=g(T)$.

Definition 2.9. Let $\left\{T, X, A, S, T_{0}\right\}$ be a hybrid dynamical system, let $x \in A$ be fixed and let $g: T \rightarrow \mathbb{R}^{+}$be the embedding mapping defined previously. Suppose that $p\left(., a, t_{0}\right) \in S$ is a motion defined on $T_{a, t_{0}}$. Let $\tilde{p}\left(., a, r_{0}\right): \mathbb{R}_{r_{0}}^{+} \rightarrow X$, where $\mathbb{R}_{r_{0}}^{+}=\left\{r \in \mathbb{R}^{+}: r \geq r_{0}\right\}$, be a function having the following properties:

(i) $r_{0}=g\left(t_{0}\right)$;

(ii) $\tilde{p}\left(r, a, r_{0}\right)=p\left(g^{-1}(r), a, t_{0}\right)$ if $r \in R_{1}=g(T)$;

(iii) $\tilde{p}\left(r, a, r_{0}\right)=x$ if $r \notin R_{1}=g(T)$.

We call $\tilde{p}\left(., a, r_{0}\right)$ the embedding of $p\left(., a, t_{0}\right)$ from $T$ to $\mathbb{R}^{+}$with respect to $x$.

Definition 2.10. Let $\left\{T, X, A, S, T_{0}\right\}$ be a hybrid dynamical system and let $x \in$ $A$. The hybrid dynamical system $\left\{\mathbb{R}^{+}, X, A, \tilde{S}, \mathbb{R}_{0}^{+}\right\}$is called the embedding of $\left\{T, X, A, S, T_{0}\right\}$ from $T$ to $\mathbb{R}^{+}$with respect to $x$, where $\mathbb{R}_{0}^{+}=g\left(T_{0}\right)$ and $\tilde{S}$ is the set of all $\tilde{p}\left(., a_{0}, r_{0}\right)$, such that $\tilde{p}\left(., a_{0}, r_{0}\right)$ is the embedding of $p\left(., a_{0}, t_{0}\right)$ with respect to $x$ and $p\left(., a_{0}, t_{0}\right) \in S$.

In view of the previous definitions, any hybrid dynamical system defined on an abstract time space $T$ can be embedded into another hybrid dynamical system defined on real time space $\mathbb{R}^{+}$.

Furthermore, it should be noted that the various stability definitions given in subsection 2.2 for general hybrid dynamical systems $\left\{T, X, A, S, T_{0}\right\}$ with invariant set $M \subset A$ translate in a natural manner to the case of dynamical systems $\left\{\mathbb{R}^{+}, X, A, \tilde{S}, \mathbb{R}_{0}^{+}\right\}$. For this is enough take the metric space $\left(\mathbb{R}^{+}, d\right)$ with usual metric $d(x, y)=|x-y|, x, y \in \mathbb{R}^{+}$and $\prec=\leq$. 
Proposition 2.1. Suppose that $\left\{T, X, A, S, T_{0}\right\}$ is a hybrid dynamical system. Let $M \subset A$ be an invariant subset for $S$, and let $x$ be any fixed point in $M$. Let $\left\{\mathbb{R}^{+}, X, A, \tilde{S}, \mathbb{R}_{0}^{+}\right\}$be the embedding of $\left\{T, X, A, S, T_{0}\right\}$ from $T$ to $\mathbb{R}^{+}$with respect to $x$. Then $M$ is also an invariant subset for system $S,(S, M)$ and $(\tilde{S}, M)$ possess identical stability properties and $S$ and $\tilde{S}$ have identical boundedness properties.

Proof. See [5].

\subsection{An example}

Consider the following problem of initial value

$$
\left\{\begin{array}{c}
\dot{x}(t)-b x(t)=0 \\
x(0)=x_{0} \geq 0
\end{array},\right.
$$

where $x \in C\left[\mathbb{R}^{+}, \mathbb{R}\right]$ and $b<0$. This differential equation determine the dynamical system $\left\{T, X, A, S, T_{0}\right\}=\left\{\mathbb{R}^{+}, \mathbb{R}^{+},\left\{x_{0}\right\}, S, 0\right\}$, with $S=\left\{p\left(., x_{0}, 0\right): \mathbb{R}^{+} \rightarrow \mathbb{R}^{+}\right\}$ such that $p\left(t, x_{0}, 0\right)=x_{0} e^{b t}$. We have the follows results:

(i) $(S,\{0\})$ is invariant, since $p\left(t, x_{0}, 0\right)=p(t, 0,0)=0 e^{b t}=0 \in\{0\}$ for all $t \in \mathbb{R}^{+}$. (ii) For every $\epsilon>0$, let $0<\delta=\epsilon$ and then $d\left(p\left(t, x_{0}, 0\right),\{0\}\right)=x_{0} e^{b t}<\delta e^{b t}=$ $\epsilon e^{b t} \leq \epsilon$, whenever $d\left(x_{0},\{0\}\right)=x_{0}<\delta$. Therefore $(S,\{0\})$ is uniformly stable.

(iii) $(S,\{0\})$ is asymptotically estable, since if $d\left(x_{0},\{0\}\right)=x_{0}<\eta<1$ then $\lim _{t \rightarrow \infty} d\left(p\left(t, x_{0}, 0\right),\{0\}\right)=\lim _{t \rightarrow \infty} p\left(t, x_{0}, 0\right)=\lim _{t \rightarrow \infty} x_{0} e^{b t}=0$.

(iv) Given $\epsilon>0$, for $\alpha>0$ and $\alpha>-b$, let $\delta=\delta(\epsilon)>0$ such that $\delta=\epsilon$. Then, $d\left(p\left(t, x_{0}, 0\right),\{0\}\right)=p\left(t, x_{0}, 0\right)=x_{0} e^{b t}<\epsilon e^{b t} \leq \epsilon e^{-\alpha t}$, whenever $d\left(x_{0},\{0\}\right)=$ $x_{0}<\delta$. Thus $(S,\{0\})$ is exponentially estable, and then uniformly asymptotically estable.

\section{Principal Lyapunov Theorems}

To follow we presented two Principal Lyapunov Theorems for the class of discontinuous dynamical systems.

Definition 3.1. We will call a dynamical system defined on $\mathbb{R}^{+}$whose motions are not continuous with respect to time a discontinuous dynamical system.

Theorem 3.1. Let $\left\{\mathbb{R}^{+},(X, d), A, S, \mathbb{R}_{0}^{+}\right\}$be a discontinuous dynamical system and let $M \subset A$ be closed. Assume that there exists a function $V: X \times \mathbb{R}^{+} \rightarrow \mathbb{R}^{+}$and functions $\psi_{1}, \psi_{2} \in K \mathbb{R}$ such that

$$
\psi_{1}(d(x, M)) \leq V(x, t) \leq \psi_{2}(d(x, M)),
$$

for all $x \in X$ and $t \in \mathbb{R}^{+}$.

(a) Assume that for every $p\left(., a, \tau_{0}^{p}\right) \in S, V\left(p\left(t, a, \tau_{0}^{p}\right), t\right)$ is continuous on $\mathbb{R}_{\tau_{0}^{p}}^{+}=$ $\left\{t \in \mathbb{R}^{+}: t \geq \tau_{0}^{p}\right\}$ except on a set of discontinuities $E_{V(p)} \subset E_{p}$, where $E_{p}=$ $\left\{\tau_{0}^{p}, \tau_{1}^{p}, \ldots: 0 \leq \tau_{0}^{p}<\tau_{1}^{p}<\ldots\right\}$ is the set of points of discontinuities of $p\left(., a, \tau_{0}^{p}\right)$. 
Also, assume that there exists a neighborhood $U \subset A$ of $M$ such that $V\left(p\left(t, a, \tau_{0}^{p}\right), t\right)$ is nonincreasing for all $a \in U$ and all $t \geq \tau_{0}^{p}$, and assume that there exists a increasing function $h \in C\left[\mathbb{R}^{+}, \mathbb{R}^{+}\right]$, with $h(0)=0$ such that

$$
V\left(p\left(t, a, \tau_{0}^{p}\right), t\right) \leq h\left(V\left(p\left(\tau_{k}^{p}, a, \tau_{0}^{p}\right), \tau_{k}^{p}\right)\right), t \in\left(\tau_{k}^{p}, \tau_{k+1}^{p}\right) .
$$

Then $(S, M)$ is invariant and uniformly stable.

(b) If in addition to the assumptions given in (a) there exists a function $\psi_{3} \in K$ defined on $\mathbb{R}^{+}$such that

$$
D V\left(p\left(\tau_{k}^{p}, a, \tau_{0}^{p}\right), \tau_{k}^{p}\right) \leq-\psi_{3}\left(d\left(p\left(\tau_{k}^{p}, a, \tau_{0}^{p}\right), M\right)\right),
$$

for all $a \in U, k \in \mathbb{N}$, where

$$
\begin{gathered}
D V\left(p\left(\tau_{k}^{p}, a, \tau_{0}^{p}\right), \tau_{k}^{p}\right):= \\
\frac{1}{\tau_{k+1}^{p}-\tau_{k}^{p}}\left[V\left(p\left(\tau_{k+1}^{p}, a, \tau_{0}^{p}\right), \tau_{k+1}^{p}\right)-V\left(p\left(\tau_{k}^{p}, a, \tau_{0}^{p}\right), \tau_{k}^{p}\right)\right]
\end{gathered}
$$

then $(S, M)$ is uniformly asymptotically stable.

Proof. (a) We will prove that $(S, M)$ is invariant. If $a \in M$, then $V\left(p\left(\tau_{0}^{p}, a, \tau_{0}^{p}\right), \tau_{0}^{p}\right)=0$ since $V\left(p\left(\tau_{0}^{p}, a, \tau_{0}^{p}\right), \tau_{0}^{p}\right)=V\left(a, \tau_{0}^{p}\right) \leq \psi_{2}(d(a, M))$ and $d(a, M)=0$. Therefore, we know that $V\left(p\left(\tau_{k}^{p}, a, \tau_{0}^{p}\right), \tau_{k}^{p}\right)=0$ for all $k \geq 0$ since $V\left(p\left(\tau_{k}^{p}, a, \tau_{0}^{p}\right), \tau_{k}^{p}\right)$ is nonincreasing for all $a \in M$ and $\tau_{k+1}^{p} \geq \tau_{0}^{p}$ and $V(X \times$ $\left.\mathbb{R}^{+}\right) \subset \mathbb{R}^{+}$. Furthermore $V\left(p\left(t, a, \tau_{0}^{p}\right), t\right)=0$ for all $t \geq \tau_{0}^{p}$ since $V\left(p\left(t, a, \tau_{0}^{p}\right), t\right) \leq$ $h\left(V\left(p\left(\tau_{k}^{p}, a, \tau_{0}^{p}\right), \tau_{k}^{p}\right)\right)=h(0)=0$. This implies that $\psi_{1}\left(d\left(p\left(t, a, \tau_{0}^{p}\right), M\right)\right) \leq$

$V\left(p\left(t, a, \tau_{0}^{p}\right), t\right)=0$ and then $d\left(p\left(t, a, \tau_{0}^{p}\right), M\right)=0$, that is, $p\left(t, a, \tau_{0}^{p}\right) \in M$ for all $t \geq \tau_{0}^{p}$, since $M$ is closed. Therefore $(S, M)$ is invariant.

Since $h$ is continuous and $h(0)=0$, then for any $\epsilon>0$ there exists $\delta=\delta(\epsilon)>0$ such that $h(y)<\psi_{1}(\epsilon)$ as long as $0 \leq y \leq \delta$. We can assume that $\delta<\psi_{1}(\epsilon)$. Thus, for any motion $p\left(., a, \tau_{0}^{p}\right) \in S$, as long as the initial condition $d(a, M)<\psi_{2}^{-1}(\delta)$ is satisfied, with $a \in U$, it follows that $V\left(p\left(\tau_{0}^{p}, a, \tau_{0}^{p}\right), \tau_{0}^{p}\right)=V\left(a, \tau_{0}^{p}\right) \leq \psi_{2}(d(a, M))<$ $\psi_{2}\left(\psi_{2}^{-1}(\delta)\right)=\delta$ and $V\left(p\left(\tau_{k}^{p}, a, \tau_{0}^{p}\right), \tau_{k}^{p}\right)<\delta$ for all $k$, since $V\left(p\left(\tau_{k}^{p}, a, \tau_{0}^{p}\right), \tau_{k}^{p}\right)$ is nonincreasing and then

$$
d\left(p\left(\tau_{k}^{p}, a, \tau_{0}^{p}\right), M\right) \leq \psi_{1}^{-1}\left(V\left(p\left(\tau_{k}^{p}, a, \tau_{0}^{p}\right), \tau_{k}^{p}\right)\right)<\psi_{1}^{-1}(\delta)<\epsilon .
$$

Furthermore, for any $t \in\left(\tau_{k}^{p}, \tau_{k+1}^{p}\right)$, we can conclude that

$$
V\left(p\left(t, a, \tau_{0}^{p}\right), t\right) \leq h\left(V\left(p\left(\tau_{k}^{p}, a, \tau_{0}^{p}\right), \tau_{k}^{p}\right)\right) \leq h(\delta)<\psi_{1}(\epsilon)
$$

and

$$
d\left(p\left(t, a, \tau_{0}^{p}\right), M\right) \leq \psi_{1}^{-1}\left(V\left(p\left(t, a, \tau_{0}^{p}\right), t\right)\right)<\psi_{1}^{-1}\left(\psi_{1}(\epsilon)\right)=\epsilon .
$$

Therefore $(S, M)$ is uniformly stable.

(b) Letting $z_{k}^{p}=V\left(p\left(\tau_{k}^{p}, a, \tau_{0}^{p}\right), \tau_{k}^{p}\right)$, with $a \in U$, we obtain from the assumptions of the theorem that

$$
-\left(\tau_{k+1}^{p}-\tau_{k}^{p}\right) \psi_{3}\left(\psi_{2}^{-1}\left(z_{k}^{p}\right)\right)=-\left(\tau_{k+1}^{p}-\tau_{k}^{p}\right) \psi_{3}\left(\psi_{2}^{-1}\left(V\left(p\left(\tau_{k}^{p}, a, \tau_{0}^{p}\right), \tau_{k}^{p}\right)\right)\right) \geq
$$




$$
\begin{gathered}
-\left(\tau_{k+1}^{p}-\tau_{k}^{p}\right) \psi_{3}\left(d\left(p\left(\tau_{k}^{p}, a, \tau_{0}^{p}\right), M\right)\right) \geq \\
V\left(p\left(\tau_{k+1}^{p}, a, \tau_{0}^{p}\right), \tau_{k+1}^{p}\right)-V\left(p\left(\tau_{k}^{p}, a, \tau_{0}^{p}\right), \tau_{k}^{p}\right)=z_{k+1}^{p}-z_{k}^{p}
\end{gathered}
$$

If we denote $\psi=\psi_{3} \circ \psi_{2}^{-1}$, then $\psi \in K$ and the last inequality becomes

$$
z_{k+1}^{p}-z_{k}^{p} \leq-\left(\tau_{k+1}^{p}-\tau_{k}^{p}\right) \psi\left(z_{k}^{p}\right) .
$$

It follows that

$$
\psi\left(z_{k}^{p}\right) \leq \frac{z_{k}^{p}-z_{k+1}^{p}}{\tau_{k+1}^{p}-\tau_{k}^{p}} \leq \frac{z_{0}^{p}-z_{k+1}^{p}}{\tau_{k+1}^{p}-\tau_{k}^{p}} \leq \frac{z_{0}^{p}}{\tau_{k+1}^{p}-\tau_{k}^{p}} .
$$

Now, consider a fixed $\delta>0$. For any $\epsilon>0$, we can choose a $\tau>0$ such that

$$
\max \left\{\psi_{1}\left(\psi^{-1}\left(\frac{\psi_{2}(\delta)}{\tau}\right)\right), \psi_{1}^{-1}\left(h\left(\psi^{-1}\left(\frac{\psi_{2}(\delta)}{\tau}\right)\right)\right)\right\}<\epsilon
$$

and $\tau_{k+1}^{p}-\tau_{k}^{p}>\tau$ for all $k$. Let $a \in U \subset A$ with $d(a, M)<\delta$ and $\tau_{0}^{p} \in \mathbb{R}_{0}^{+}$any. For any $t \geq \tau_{0}^{p}+\tau, t$ must belong to some interval $\left[\tau_{k}^{p}, \tau_{k+1}^{p}\right)$ for some $k$. It follows from (3.2) that

$$
\begin{gathered}
\psi\left(z_{k}^{p}\right) \leq \frac{z_{0}^{p}}{\tau_{k+1}^{p}-\tau_{k}^{p}}=\frac{V\left(p\left(\tau_{0}^{p}, a, \tau_{0}^{p}\right), \tau_{0}^{p}\right)}{\tau_{k+1}^{p}-\tau_{k}^{p}}=\frac{V\left(a, \tau_{0}^{p}\right)}{\tau_{k+1}^{p}-\tau_{k}^{p}}< \\
<\frac{V\left(a, \tau_{0}^{p}\right)}{\tau} \leq \frac{\psi_{2}(d(a, M))}{\tau}<\frac{\psi_{2}(\delta)}{\tau}
\end{gathered}
$$

which implies that

$$
z_{k}^{p}=V\left(p\left(\tau_{k}^{p}, a, \tau_{0}^{p}\right), \tau_{k}^{p}\right)<\psi^{-1}\left(\frac{\psi_{2}(\delta)}{\tau}\right)
$$

and

$$
V\left(p\left(t, a, \tau_{0}^{p}\right), t\right) \leq h\left(V\left(p\left(\tau_{k}^{p}, a, \tau_{0}^{p}\right), \tau_{k}^{p}\right)\right) \leq h\left(\psi^{-1}\left(\frac{\psi_{2}(\delta)}{\tau}\right)\right)
$$

if $t \in\left(\tau_{k}^{p}, \tau_{k+1}^{p}\right)$. In the case when $t=\tau_{k}^{p}$, it follows from (3.3) that

$$
d\left(p\left(\tau_{k}^{p}, a, \tau_{0}^{p}\right), M\right) \leq \psi_{1}^{-1}\left(V\left(p\left(\tau_{k}^{p}, a, \tau_{0}^{p}\right), \tau_{k}^{p}\right)\right)<\psi_{1}^{-1}\left(\psi^{-1}\left(\frac{\psi_{2}(\delta)}{\tau}\right)\right)<\epsilon .
$$

In the case when $t \in\left(\tau_{k}^{p}, \tau_{k+1}^{p}\right)$, we can conclude from (3.4) that

$$
d\left(p\left(t, a, \tau_{0}^{p}\right), M\right) \leq \psi_{1}^{-1}\left(V\left(p\left(t, a, \tau_{0}^{p}\right), t\right)\right) \leq \psi_{1}^{-1}\left(h\left(\psi^{-1}\left(\frac{\psi_{2}(\delta)}{\tau}\right)\right)\right)<\epsilon .
$$

This prove that $(S, M)$ is uniformly asymptotically stable.

Theorem 3.2. Let $\left\{\mathbb{R}^{+},(X, d), A, S, \mathbb{R}_{0}^{+}\right\}$be a discontinuous dynamical system and let $M \subset A$ be closed. Assume that there exists a function $V: X \times \mathbb{R}^{+} \rightarrow \mathbb{R}^{+}$and positive constants $c_{1}, c_{2}$ and $b$ such that

$$
c_{1}(d(x, M))^{b} \leq V(x, t) \leq c_{2}(d(x, M))^{b},
$$


for all $x$ in some neighborhood $X_{1}$ of $M$ and $t \in \mathbb{R}^{+}$.

(i) Assume that for every $p\left(., a, \tau_{0}^{p}\right) \in S, V\left(p\left(t, a, \tau_{0}^{p}\right), t\right)$ is continuous on $\mathbb{R}_{\tau_{0}^{p}}^{+}=$ $\left\{t \in \mathbb{R}^{+}: t \geq \tau_{0}^{p}\right\}$ except on a set of discontinuities $E_{V(p)} \subset E_{p}$, where $E_{p}=$ $\left\{\tau_{0}^{p}, \tau_{1}^{p}, \ldots: 0 \leq \tau_{0}^{p}<\tau_{1}^{p}<\ldots\right\}$ is the set of points of discontinuities of $p\left(., a, \tau_{0}^{p}\right)$. Moreover, assume that there exists a function $h \in C\left[\mathbb{R}^{+}, \mathbb{R}^{+}\right]$, with $h(0)=0$ such that

$$
V\left(p\left(t, a, \tau_{0}^{p}\right), t\right) \leq h\left(V\left(p\left(\tau_{k}^{p}, a, \tau_{0}^{p}\right), \tau_{k}^{p}\right)\right),
$$

for $t \in\left(\tau_{k}^{p}, \tau_{k+1}^{p}\right)$, and such that for some positive constant $q$, $h$ satisfies

$$
\lim _{r \rightarrow 0} \frac{h(r)}{|r|^{q}}=0
$$

(ii) Suppose that there exists a constant $c_{3}>0$ such that

$$
D V\left(p\left(\tau_{k}^{p}, a, \tau_{0}^{p}\right), \tau_{k}^{p}\right) \leq-c_{3}\left[d\left(p\left(\tau_{k}^{p}, a, \tau_{0}^{p}\right), M\right)\right]^{b},
$$

for all $a \in X_{1}$ and $k \in \mathbb{N}$, where $D V\left(p\left(\tau_{k}^{p}, a, \tau_{0}^{p}\right), \tau_{k}^{p}\right)$ is defined in (3.1).

Then $(S, M)$ is exponentially stable.

Proof. See [5].

\section{Converse Theorem}

Now we presented a converse theorem of the theorem 3.2 in the following sense:

Theorem 4.1. Let $\left\{\mathbb{R}^{+},(X, d), A, S, \mathbb{R}_{0}^{+}=\mathbb{R}^{+}\right\}$be a discontinuous dynamical system and let $M \subset A$ be a closed invariant set, where $A$ is a neighborhood of $M$. Suppose that:

(i) every $p\left(., a, \tau_{0}^{p}\right) \in S$ is continuous everywhere on $\left[\tau_{0}^{p}, \infty\right)$, except on a set $E_{p}=\left\{\tau_{0}^{p}, \tau_{1}^{p}, \ldots: \tau_{0}^{p}<\tau_{1}^{p}<\ldots\right\}$, being $l=\inf _{p \in S}\left\{\tau_{k+1}^{p}-\tau_{k}^{p}\right\}>0$ and $L=$ $\sup _{p \in S}\left\{\tau_{k+1}^{p}-\tau_{k}^{p}\right\}<\infty$;

(ii) for any $p\left(., a, \tau_{0}^{p}\right) \in S$ is true that $p\left(t^{\prime}, p\left(t, a, \tau_{0}^{p}\right), t\right)=p\left(t^{\prime}, a, \tau_{0}^{p}\right)$ for all $t \in \mathbb{R}_{0}^{+}$ and $t^{\prime} \geq t$. Furthermore, $p\left(t^{\prime}, a, t\right) \notin M$ if a $\notin M$, therefore $d\left(p\left(t^{\prime}, a, t\right), M\right)>0$ for all $t^{\prime} \geq t$ if $a \notin M$, since $M$ is closed.

Let $(S, M)$ be exponentially stable. Then there exists a neighborhood $X_{1}$ of $M$ such that $X_{1} \subset A$, and a mapping $V: X_{1} \times \mathbb{R}^{+} \rightarrow \mathbb{R}^{+}$which satisfies the following conditions:

(a)there exist $\psi_{1}, \psi_{2} \in K$ defined on $\mathbb{R}^{+}$such that

$$
\psi_{1}(d(x, M)) \leq V(x, t) \leq \psi_{2}(d(x, M)),
$$

for all $(x, t) \in X_{1} \times \mathbb{R}^{+}$.

(b) there exists a constant $c>0$ such that for every $p\left(., a, \tau_{0}^{p}\right) \in S$,

$$
D V\left(p\left(\tau_{k}^{p}, a, \tau_{0}^{p}\right), \tau_{k}^{p}\right) \leq-c V\left(p\left(\tau_{k}^{p}, a, \tau_{0}^{p}\right), \tau_{k}^{p}\right),
$$


for $k \in \mathbb{N}$, where $a \in X_{1}$.

(c) there exists a function $h \in C\left[\mathbb{R}^{+}, \mathbb{R}^{+}\right]$, with $h(0)=0$ and $\lim _{\theta \rightarrow 0^{+}} \frac{h(\theta)}{\theta^{q}}=0$ for some constant $q>0$, such that

$$
V\left(p\left(t, a, \tau_{0}^{p}\right), t\right) \leq h\left(V\left(p\left(\tau_{k}^{p}, a, \tau_{0}^{p}\right), \tau_{k}^{p}\right),\right.
$$

for every $p\left(., a, \tau_{0}^{p}\right) \in S, t \in\left(\tau_{k}^{p}, \tau_{k+1}^{p}\right), a \in X_{1}$ and $\tau_{0}^{p} \in \mathbb{R}^{+}$.

Proof. Since $(S, M)$ exponentially stable, for every $\epsilon>0$ there exists $\alpha>0$ and $r_{0}=$ $r_{0}(\epsilon)$ such that $d\left(p\left(t, a, \tau_{0}^{p}\right), M\right)<\epsilon e^{-\alpha\left(t-\tau_{0}^{p}\right)}$ for all $p\left(., a, \tau_{0}^{p}\right) \in S$ and all $t \in T_{a, \tau_{0}^{p}}$, whenever $d(a, M)<r_{0}$. Let $\phi \in K$ defined on $\left[0, r_{0}\right]$ such that $\phi(d(a, M)) \geq \epsilon$ if $a \notin M$, then $d\left(p\left(t, a, \tau_{0}^{p}\right), M\right)<\epsilon e^{-\alpha\left(t-\tau_{0}^{p}\right)} \leq \phi(d(a, M)) e^{-\alpha\left(t-\tau_{0}^{p}\right)}$. If $a \in M$, $p\left(t, a, \tau_{0}^{p}\right) \in M$ therefore $d\left(p\left(t, a, \tau_{0}^{p}\right), M\right)=0 \leq \phi(d(a, M)) e^{-\alpha\left(t-\tau_{0}^{p}\right)}=0$, thus

$$
d\left(p\left(t, a, \tau_{0}^{p}\right), M\right) \leq \phi(d(a, M)) e^{-\alpha\left(t-\tau_{0}^{p}\right)} .
$$

Let $X_{1}=\left\{x \in A: d(x, M)<r_{0}\right\}$. For $(x, t) \in X_{1} \times \mathbb{R}^{+}$, define

$$
V(x, t)=\sup _{t^{\prime} \geq t}\left\{d\left(p\left(t^{\prime}, x, t\right), M\right) \cdot e^{\alpha\left(t^{\prime}-t\right)}\right\} .
$$

For $a \in X_{1}$ and $\tau_{0}^{p} \in \mathbb{R}^{+}$, we have

$$
\begin{gathered}
V\left(p\left(t, a, \tau_{0}^{p}\right), t\right)=\sup _{t^{\prime} \geq t}\left\{d\left(p\left(t^{\prime}, p\left(t, a, \tau_{0}^{p}\right), t\right), M\right) \cdot e^{\alpha\left(t^{\prime}-t\right)}\right\} \\
=\sup _{t^{\prime} \geq t}\left\{d\left(p\left(t^{\prime}, a, \tau_{0}^{p}\right), M\right) \cdot e^{\alpha\left(t^{\prime}-t\right)}\right\}
\end{gathered}
$$

therefore

$$
\begin{gathered}
V\left(p\left(\tau_{k+1}^{p}, a, \tau_{0}^{p}\right), \tau_{k+1}^{p}\right)=\sup _{t^{\prime} \geq \tau_{k+1}^{p}}\left\{d\left(p\left(t^{\prime}, a, \tau_{0}^{p}\right), M\right) e^{\alpha\left(t^{\prime}-\tau_{k+1}^{p}\right)}\right\} \\
=\sup _{t^{\prime} \geq \tau_{k+1}^{p}}\left\{d\left(p\left(t^{\prime}, a, \tau_{0}^{p}\right), M\right) e^{\alpha\left(t^{\prime}-\tau_{k}^{p}\right)} e^{-\alpha\left(\tau_{k+1}^{p}-\tau_{k}^{p}\right)}\right\} \\
=e^{-\alpha\left(\tau_{k+1}^{p}-\tau_{k}^{p}\right)} \sup _{t^{\prime} \geq \tau_{k+1}^{p}}\left\{d\left(p\left(t^{\prime}, a, \tau_{0}^{p}\right), M\right) e^{\alpha\left(t^{\prime}-\tau_{k}^{p}\right)}\right\} \\
\leq e^{-\alpha l} \sup _{t^{\prime} \geq \tau_{k+1}^{p}}\left\{d\left(p\left(t^{\prime}, a, \tau_{0}^{p}\right), M\right) e^{\alpha\left(t^{\prime}-\tau_{k}^{p}\right)}\right\} \\
\leq e^{-\alpha l} \sup _{t^{\prime} \geq \tau_{k}^{p}}\left\{d\left(p\left(t^{\prime}, a, \tau_{0}^{p}\right), M\right) e^{\alpha\left(t^{\prime}-\tau_{k}^{p}\right)}\right\}=e^{-\alpha l} V\left(p\left(\tau_{k}^{p}, a, \tau_{0}^{p}\right), \tau_{k}^{p}\right) .
\end{gathered}
$$

Letting $c=(1 / L)\left(1-e^{-\alpha l}\right)$, we obtain

$$
\begin{gathered}
D V\left(p\left(\tau_{k}^{p}, a, \tau_{0}^{p}\right), \tau_{k}^{p}\right)=\frac{1}{\tau_{k+1}^{p}-\tau_{k}^{p}}\left(V\left(p\left(\tau_{k+1}^{p}, a, \tau_{0}^{p}\right), \tau_{k+1}^{p}\right)-V\left(p\left(\tau_{k}^{p}, a, \tau_{0}^{p}\right), \tau_{k}^{p}\right)\right) \\
\leq-\frac{1}{\tau_{k+1}^{p}-\tau_{k}^{p}}\left(1-e^{-\alpha l}\right) V\left(p\left(\tau_{k}^{p}, a, \tau_{0}^{p}\right), \tau_{k}^{p}\right)
\end{gathered}
$$




$$
\leq-\frac{1}{L}\left(1-e^{-\alpha l}\right) V\left(p\left(\tau_{k}^{p}, a, \tau_{0}^{p}\right), \tau_{k}^{p}\right)=-c V\left(p\left(\tau_{k}^{p}, a, \tau_{0}^{p}\right), \tau_{k}^{p}\right) .
$$

Also, for all $(x, t) \in X_{1} \times \mathbb{R}^{+}$(4.1) imply that

$$
\begin{gathered}
V(x, t)=\sup _{t^{\prime} \geq t}\left\{d\left(p\left(t^{\prime}, x, t\right), M\right) e^{\alpha\left(t^{\prime}-t\right)}\right\} \\
\left.\leq \sup _{t^{\prime} \geq t}\left\{\phi(d(x, M)) e^{-\alpha\left(t^{\prime}-t\right)} e^{\alpha\left(t^{\prime}-t\right)}\right)\right\}=\sup _{t^{\prime} \geq t}\{\phi(d(x, M))\}=\phi(d(x, M)) .
\end{gathered}
$$

Letting $\psi_{2} \in K$ defined on $\mathbb{R}^{+}$such that $\psi_{2}(r)=\phi(r)$ if $r \in\left[0, r_{0}\right]$, then

$$
V(x, t) \leq \psi_{2}(d(x, M)) .
$$

Furthermore

$$
V(x, t)=\sup _{t^{\prime} \geq t}\left\{d\left(p\left(t^{\prime}, x, t\right), M\right) e^{\alpha\left(t^{\prime}-t\right)}\right\} \geq d\left(p\left(t^{\prime}, x, t\right), M\right) \geq \psi_{1}(d(x, M))
$$

for some $\psi_{1} \in K$ defined on $\mathbb{R}^{+}$. By (4.2) we have for every $t \in\left(\tau_{k}^{p}, \tau_{k+1}^{p}\right), \tau_{0}^{p} \in \mathbb{R}^{+}$ and $a \in X_{1}$

$$
\begin{gathered}
V\left(p\left(t, a, \tau_{0}^{p}\right), t\right)=\sup _{t^{\prime} \geq t}\left\{d\left(p\left(t^{\prime}, a, \tau_{0}^{p}\right), M\right) e^{\alpha\left(t^{\prime}-t\right)}\right\} \\
=\sup _{t^{\prime} \geq t}\left\{d\left(p\left(t^{\prime}, a, \tau_{0}^{p}\right), M\right) e^{\alpha\left(t^{\prime}-\tau_{k}^{p}\right)} e^{-\alpha\left(t-\tau_{k}^{p}\right)}\right\} \leq \sup _{t^{\prime} \geq t}\left\{d\left(p\left(t^{\prime}, a, \tau_{0}^{p}\right), M\right) e^{\alpha\left(t^{\prime}-\tau_{k}^{p}\right)}\right\} \\
\leq \sup _{t^{\prime} \geq \tau_{k}^{p}}\left\{d\left(p\left(t^{\prime}, a, \tau_{0}^{p}\right), M\right) e^{\alpha\left(t^{\prime}-\tau_{k}^{p}\right)}\right\}=V\left(p\left(\tau_{k}^{p}, a, \tau_{0}^{p}\right), \tau_{k}^{p}\right) .
\end{gathered}
$$

Letting $h \in C\left[\mathbb{R}^{+}, \mathbb{R}^{+}\right]$such that $h(r)=r$ and $q=\frac{1}{2}$, it follows that $\lim _{\theta \rightarrow 0^{+}} \frac{h(\theta)}{\theta^{q}}=$ $\frac{\theta}{\theta^{\frac{1}{2}}}=0$ and

$$
V\left(p\left(t, a, \tau_{0}^{p}\right), t\right) \leq V\left(p\left(\tau_{k}^{p}, a, \tau_{0}^{p}\right), \tau_{k}^{p}\right)=h\left(V\left(p\left(\tau_{k}^{p}, a, \tau_{0}^{p}\right), \tau_{k}^{p}\right)\right)
$$

Resumo. Neste trabalho introduzimos um modelo geral para os Sistemas Dinâmicos Híbridos e para tais sistemas introduzimos o conceito usual de estabilidade de Lyapunov. Além disso, estabelecemos dois Teoremas Principais de Lyapunov e um teorema de conversão.

\section{References}

[1] L. Hou, "Qualitative Analysis of Discontinuous Deterministic and Stochastic Dynamical Systems", Ph.D. Thesis, University of Notre Dame, Indiana, 2000.

[2] A.N. Michel, Recent trends in the stability analysis of hybrid dynamical systems, IEEE Transactions on Circuits and Systems, 45, No.1 (1999), 120-134. 
[3] A.N. Michel, L. Hou, Stability analysis of a general class of hybrid dynamical systems, in "Proceedings of the American Control Conference", pp. 2805-2809, Albuquerque, New Mexico, 1997.

[4] A.N. Michel, B. Hu, Towards a stability theory of general hybrid dynamical systems, Automatica, 35 (1999), 371-384.

[5] A.N. Michel, B. Hu, K. Wang, "Qualitative Theory of Dynamical Systems", Marcel Dekker, New York, 2001.

[6] H. Ye, A.N. Michel, Stability theory for hybrid dynamical systems, IEEE Transactions on Automatic Control, 43, No.4 (1998), 461-474.

[7] H. Ye, A.N. Michel, A general model for the qualitative analysis of hybrid dynamical systems, in "Proceedings of the 34th Conference on Decision and Control", pp. 1473-1477, IEEE, New Orleans, 1995. 